Europhys. Lett., 68 (3), pp. 405-411 (2004)

DOI: $10.1209 / \mathrm{epl} / \mathrm{i} 2004-10201-\mathrm{y}$

\title{
Twinning pathway in BCC molybdenum
}

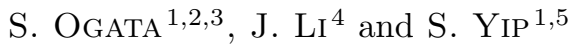 \\ 1 Department of Nuclear Engineering, Massachusetts Institute of Technology \\ Cambridge, MA 02139, USA \\ 2 Handai Frontier Research Center, Osaka University - Osaka, Japan \\ 3 Department of Mechanical Engineering and Systems, Osaka University \\ Osaka 565-0871, Japan \\ 4 Department of Materials Science and Engineering, Ohio State University \\ Columbus, OH 43210, USA \\ 5 Department of Materials Science and Engineering \\ Massachusetts Institute of Technology - Cambridge, MA 02139, USA
}

received 27 May 2004; accepted in final form 27 August 2004 published online 16 October 2004

PACS. 62.20.Fe - Deformation and plasticity (including yield, ductility, and superplasticity). PACS. 61.72.Nn - Stacking faults and other planar or extended defects.

PACS. 61.72.Mm - Grain and twin boundaries.

\begin{abstract}
The $(2 \overline{1} \overline{1})\langle 111\rangle$ twinning energy landscape of BCC Mo is determined using the density functional theory for embryos containing 2 to 7 layers. The 2-layer embryo is metastable, whereas the 3- and 4-layer ones are unstable. Layer-by-layer growth starts at 5 layers. The twin boundary formation and migration energies are found to be $607 \mathrm{~mJ} / \mathrm{m}^{2}$ and $40 \mathrm{~mJ} / \mathrm{m}^{2}$, respectively, indicating that twin partial dislocations have wide cores and high mobilities. The stress to homogeneously nucleate an additional partial loop on the boundary of a sufficiently thick twin is only $1.4 \mathrm{GPa}$; this implies that once a deformation twin reaches critical thickness, which we estimate to be 6 layers, subsequent growth in thickness is easy.
\end{abstract}

Deformation twinning [1] is a primary mode of strain-energy relaxation [2] which competes with dislocation slip as the dominant carrier of plastic deformation. The outcome of the competition depends on the stress level [3], temperature, nature of existing defects like cracks [4], and intrinsic material properties [5]. It is generally believed that at low-to-intermediate stress levels, the activation energy of the nucleating deformation twin is greater than that of the slip. However, once nucleated, a deformation twin is able to produce a large amount of plastic strain within a very short time. Therefore, deformation twinning tends to occur more frequently under high strain-rate loading such as laser shock [6], at lower temperatures [7], and near stress concentrators $[2,8]$. The mesoscopic kinematics of twin growth has been proposed to involve the pole mechanism [9] and variants $[10,11]$, and the double-cross-slip mechanism $[12,13]$. On the other hand, microscopic energetic information, even for simple metals, is lacking.

Recently, Tadmor and collaborators have established an intrinsic material property called the "twinnability" [4], which is the ratio of $\gamma_{\mathrm{us}}$, the unstable stacking energy, to $\gamma_{\mathrm{ut}}$, the unstable twinning energy, both in $\mathrm{J} / \mathrm{m}^{2}$. Basically, $\gamma_{\mathrm{us}}$ and $\gamma_{\mathrm{ut}}$ are the barriers for a 1-layer partial (c) EDP Sciences 


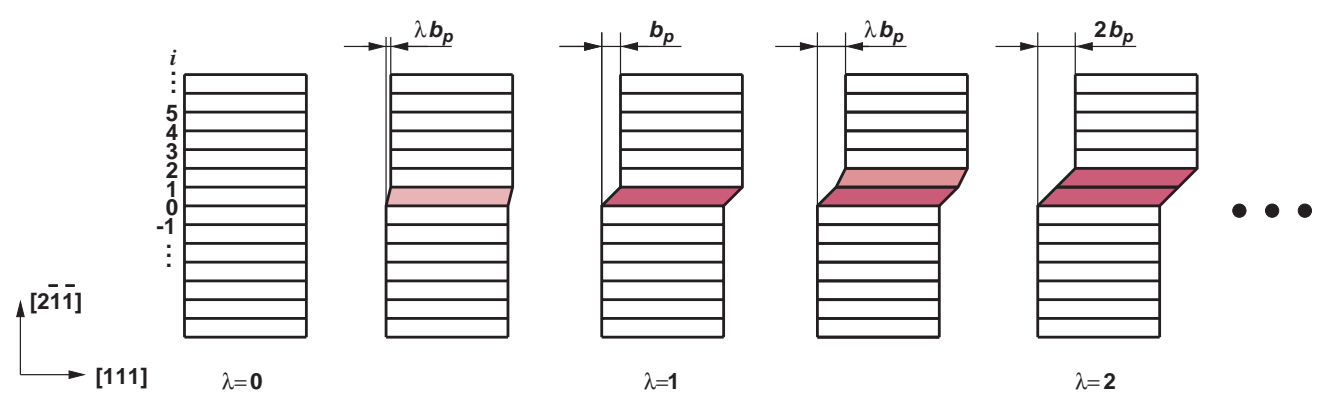

Fig. 1 - Schematic illustration of the twinning pathway. See the text for detail.

fault to become a 1-layer full fault (with zero energy) and a 2-layer partial fault, respectively. The claim that $\gamma_{\text {us }} / \gamma_{\text {ut }}$ fully characterizes twinnability implicitly assumes that a 2-layer partial fault behaves as a mature twin embryo in that the evolution of 3-layer, 4-layer, ... partial faults follows in the same manner as the 1-layer to 2-layer transition. While this may be true for simple FCC metals, we find that this is not the case for BCC metals on the basis of density functional theory (DFT) calculations. Along the twinning pathway of BCC molybdenum, the fault energy does not show a steady-state oscillatory pattern until $n=6$ layers.

The generalized stacking fault (GSF) energy established by Frenkel [14] and Vitek [15,16] is an important material concept in the theory of crystalline defects. Its most general form is an energy function $E\left(\left\{\Delta \boldsymbol{x}_{i}\right\}\right)$, where integer $i \in-\infty \ldots \infty$ labels sequential atomic stacking planes, and $\Delta \boldsymbol{x}_{i}$ is the relative shear displacement between plane $i$ and $i-1$. By definition, $E\left(\left\{\Delta \boldsymbol{x}_{i}\right\}\right)=0$ when all $\Delta \boldsymbol{x}_{i}$ 's are zero, and clearly, $E\left(\left\{\Delta \boldsymbol{x}_{i}\right\}\right)=E\left(\left\{\Delta \boldsymbol{x}_{i}+n_{i} \boldsymbol{b}\right\}\right)$, i.e., it is periodic in any displacement with period $\boldsymbol{b}$, the full Burgers vector $\left(\boldsymbol{b}=[111] a_{0} / 2\right.$ in BCC and $[01 \overline{1}] a_{0} / 2$ in FCC crystals).

Two aspects of $E\left(\left\{\Delta \boldsymbol{x}_{i}\right\}\right)$ in FCC metals have been probed using electronic-structure calculations [5,17]. One is to consider a family of functions $\gamma_{i}(\boldsymbol{x}) \equiv E\left(\left\{\Delta \boldsymbol{x}_{i}\right\}\right) / N S_{0}$, with $\Delta \boldsymbol{x}_{1}=\Delta \boldsymbol{x}_{2}=\ldots=\Delta \boldsymbol{x}_{N}=\boldsymbol{x}$, all other $\Delta \boldsymbol{x}_{i}$ 's zero, and $S_{0}$ the cross-sectional area, and study the asymptotic approach of $\gamma_{N}(\boldsymbol{x})$ to $\gamma_{\infty}(\boldsymbol{x})$, the affine strain-energy landscape. This turns out to be a good measure of directional bonding [17]. The other aspect is to examine $E\left(\left\{\Delta \boldsymbol{x}_{i}\right\}\right)$ on the so-called twinning pathway, $\gamma_{\mathrm{t}}(\lambda>0) \equiv E\left(\left\{\Delta \boldsymbol{x}_{i}\right\}\right) / S_{0}$, with $\Delta \boldsymbol{x}_{i \geq 1}=((\lambda-i+1) H(\lambda-i+1)-(\lambda-i) H(\lambda-i)) \boldsymbol{b}_{\mathrm{p}}, \Delta \boldsymbol{x}_{i \leq 0}=0, \boldsymbol{b}_{\mathrm{p}}$ is the partial Burgers vector of the twinning system $\left(\boldsymbol{b}_{\mathrm{p}}=[111] a_{0} / 6\right.$ in BCC and $[11 \overline{2}] a_{0} / 6$ in FCC), and $H(x)$ is the Heaviside step function (see fig. 1). In FCC metals, the competition between full dislocation nucleation and twin nucleation has been shown to be governed by the values of saddle energies on $\gamma_{1}\left(\lambda \boldsymbol{b}_{\mathrm{p}}\right)$ and $\gamma_{\mathrm{t}}(\lambda)$, respectively [4]. $\gamma_{\mathrm{t}}(\lambda)$ of FCC metals is relatively simple, possessing a metastable minimum at every $\lambda=n$ for $n=1,2,3, \ldots$ and converging to a steady oscillatory pattern for $\lambda \geq n=2$ [18]. In this work, we show that BCC Mo possesses a much more complicated twinning energy pathway, one which indicates long-ranged mechanical coupling, with $n=1,3$ and 4 metastable minima missing, and does not converge to a steady oscillatory pattern until $\lambda \geq n=6$. This further enriches the scenario of dislocation-twinning competition in BCC metals, which occurs more frequently than in FCC metals.

We use the Vienna ab initio Simulation Package (VASP) [19] with Perdew-Wang generalized gradient approximation (GGA) [20] exchange-correlation density functional and ultrasoft (US) pseudopotential [21]. The supercell is $\boldsymbol{e}_{1} \times \boldsymbol{e}_{2} \times m \boldsymbol{e}_{3}$, with $\boldsymbol{e}_{1} \equiv[111] a_{0} / 2, \boldsymbol{e}_{2} \equiv[0 \overline{1} 1] a_{0}$, and $\boldsymbol{e}_{3} \equiv[2 \overline{1} \overline{1}] a_{0}$, and 6 atoms per $\boldsymbol{e}_{1} \times \boldsymbol{e}_{2} \times \boldsymbol{e}_{3}$. Brillouin zone (BZ) $\boldsymbol{k}$-point sampling is per- 
TABLE I - Equilibrium lattice constants $\left(a_{0}\right)$, relaxed $\left(G_{\mathrm{r}}\right)$ and unrelaxed $\left(G_{\mathrm{u}}\right)$ shear moduli along $(2 \overline{1} \overline{1})\langle 111\rangle$, and bulk modulus $(B)$ of BCC Mo.

\begin{tabular}{lcccc}
\hline & $a_{0}(\AA)$ & $G_{\mathrm{r}}(\mathrm{GPa})$ & $G_{\mathrm{u}}(\mathrm{GPa})$ & $B(\mathrm{GPa})$ \\
\hline Calc. & 3.15 & 126.8 & 134.1 & 244 \\
Expt. & $3.14[23]$ & 138.7 & 142.8 & $264[23]$ \\
\hline
\end{tabular}

formed using the Monkhorst-Pack algorithm. BZ integration follows the Methfessel-Paxton scheme [22] with the smearing width chosen so the "-TS" term is less than $0.5 \mathrm{meV} /$ atom. We use $233 \mathrm{eV}$ plane-wave energy cutoff throughout the calculations.

As benchmark, the perfect-crystal properties are computed first. In table I, the equilibrium lattice constant $\left(a_{0}\right)$ and bulk modulus $(B)$ are compared with experimental results. We then perform direct affine shear in $(2 \overline{1} \overline{1})\langle 111\rangle$ with the 5 subsidiary stress components all relaxed and unrelaxed, respectively, to determine the relaxed $\left(G_{\mathrm{r}}\right)$ and unrelaxed $\left(G_{\mathrm{u}}\right)$ shear moduli. Experimental values of $G_{\mathrm{r}}$ and $G_{\mathrm{u}}$ are tabulated using analytical formulas and experimentally measured $C_{11}, C_{12}$ and $C_{44}$. The agreement, to within $10 \%$, is considered satisfactory.

We then perform large affine shear, both relaxed and unrelaxed, in $(2 \overline{1} \overline{1})\langle 111\rangle$. In table II, the ideal shear strains and stresses obtained, defined by the point of maximum stress in the stress-strain response [17], are shown. These results agree well with the first-principles calculations reported by Krenn et al. [24].

Having probed the $\gamma_{\infty}(\boldsymbol{x})$ of $(2 \overline{1} \overline{1})\langle 111\rangle$, we now study in detail the $\gamma_{t}(\lambda)$ pathway on $(2 \overline{1} \overline{1})\langle 111\rangle$. First, we determine the asymptotic behavior of $\gamma_{t}(\lambda)$ when $\lambda \rightarrow \infty$, i.e., when the twin is very thick and the two twin boundaries are well separated. We expect a steady-state oscillatory behavior:

$$
\gamma_{\mathrm{t}}(\lambda) \longrightarrow 2 \gamma_{\mathrm{tbf}}+\gamma_{\mathrm{tb} \infty}(\lambda), \quad \text { as } \lambda \longrightarrow \infty,
$$

in which $\gamma_{\mathrm{tbf}}$ is interpreted as the twin boundary formation energy (unrelaxed), and $\gamma_{\mathrm{tb} \infty}(\lambda)$ the steady-state twin boundary migration energy profile, which is a periodic function in $\lambda$ with period 1 and with $\gamma_{\mathrm{tb} \infty}(\lambda=0)=0$. We then define $\gamma_{\mathrm{tbm}} \equiv \max _{\lambda} \gamma_{\mathrm{tb} \infty}(\lambda)$ as the twin boundary migration energy. It is the energy barrier per area for a very thick twin to have one of its boundaries extend further by one layer: $\lambda \rightarrow \lambda+1$. In reality, this is accomplished by an additional twin partial dislocation with Burgers vector $\boldsymbol{b}_{\mathrm{p}}=[111] a_{0} / 6$ sweeping a face of the twin. According to the Peierls-Nabarro model [25-27], the $\gamma_{\mathrm{tb} \infty}(\lambda)$ profile controls the width and mobility of the twin partial dislocation. Therefore, it is important to obtain the asymptotic characteristics of $\gamma_{t}(\lambda)$.

To study the behavior of a single twin boundary, we introduce a slab model with 24 layers of Mo ( $6 \boldsymbol{e}_{3}$ supercell, $2 \boldsymbol{e}_{3}$ of which is vacuum), and with $33 \times 21 \times 1 \boldsymbol{k}$-point sampling. No vertical or in-plane relaxation of the atoms in excess of the designated shear displacements is allowed. The energy of the slab without any twin is evaluated first as a reference. Then we introduce a configuration whereby layers 1-12 are twinned with respect to layers $13-24$. The energy change with respect to the reference is $607 \mathrm{~mJ} / \mathrm{m}^{2}$, which we designate as $\gamma_{\text {tbf }}$.

We then slide layers 13-24 as a rigid block along $\boldsymbol{b}_{\mathrm{p}}$, such that the final configuration has layers $1-13$ twinned. The energy profile along this path is plotted in fig. 2(a), with the energy

TABLE II - Ideal shear strains and stresses of BCC Mo along $(2 \overline{1} \overline{1})\langle 111\rangle$.

\begin{tabular}{|c|c|c|c|c|c|}
\hline \multicolumn{3}{|c|}{ Relaxed } & \multicolumn{3}{|c|}{ Unrelaxed } \\
\hline$\gamma_{\mathrm{m}}^{\mathrm{r}}$ & $\sigma_{\mathrm{m}}^{\mathrm{r}}(\mathrm{GPa})$ & $\sigma_{\mathrm{m}}^{\mathrm{r}} / G_{\mathrm{r}}$ & $\gamma_{\mathrm{m}}^{\mathrm{u}}$ & $\sigma_{\mathrm{m}}^{\mathrm{u}}(\mathrm{GPa})$ & $\sigma_{\mathrm{m}}^{\mathrm{u}} / G_{\mathrm{u}}$ \\
\hline 0.175 & 14.84 & 0.117 & 0.177 & 15.99 & 0.119 \\
\hline
\end{tabular}



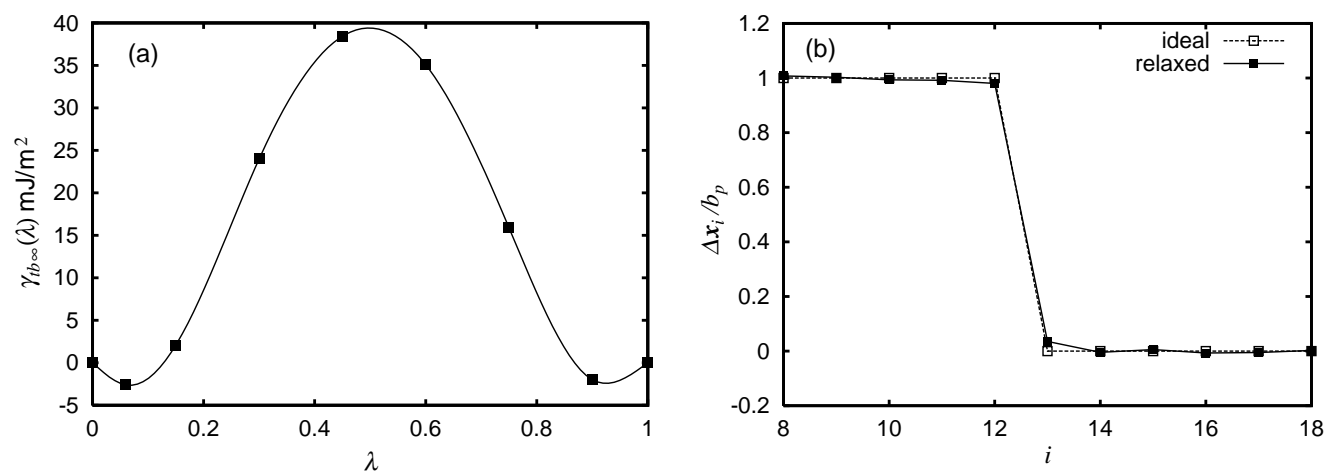

Fig. 2 - (a) Energy landscape of twin migration in Mo. Initially a twin boundary is introduced at the center of a 24-layer slab model, and then the twin boundary is shifted by 1 layer by sliding the upper half. The twin boundary migration energy is estimated as $40 \mathrm{~mJ} / \mathrm{m}^{2}$. The twin boundary formation energy, which can be estimated from the energy difference between perfect and twinned slabs, is estimated to be $607 \mathrm{~mJ} / \mathrm{m}^{2}$. (b) Layer displacements (normalized by $\boldsymbol{b}_{\mathrm{p}}$ ) near the ideal and relaxed twin boundaries.

at the origin set to be zero. No vertical or in-plane relaxation has been allowed. $\gamma_{\text {tbm }}$ can be estimated from this plot to be $40 \mathrm{~mJ} / \mathrm{m}^{2}$. If vertical relaxation is allowed, $\gamma_{\mathrm{tbm}}$ can be expected to be reduced, although not significantly.

A feature of fig. 2(a) is that the slope at 0 is not exactly zero. This suggests there is a minute in-plane force on layer 13 adjacent to the sharp (ideal) twin boundary, indicating a slight tendency of the boundary to broaden. This was suggested by Mrovec et al. [28]. To explore the extent of this effect, we then allow all 24 layers to freely relax in the in-plane directions, until the energy change between two consecutive ionic steps is less than $1 \mathrm{meV}$. The unrelaxed and relaxed displacements of layers 8-17 are shown in fig. 2(b), respectively. We see there is a small relaxation effect, but overall the twin boundary remains very sharp and is a locally stable energy minimum. This partially relaxed twin boundary has energy $580 \mathrm{~mJ} / \mathrm{m}^{2}$.

With the large- $\lambda$ asymptotic behavior of $\gamma_{\mathrm{t}}(\lambda)$ estimated, we then compute $\gamma_{\mathrm{t}}(\lambda)$ at smaller $\lambda$. A different setup is used. Previously, the 24 layers form a twinned-untwinned bicrystal in the supercell. Now, the 24 layers form an untwinned-twinned-untwinned sandwich, with the twinned regions at the center and as far from the surface as possible. Unrelaxed, rigidblock sliding is carried out for each episode of $n<\lambda<n+1$, that enables the deformation twin to grow by one layer. And then sliding is initiated again in the next layer. The same $33 \times 21 \times 1 \boldsymbol{k}$-point sampling is used, which is found to give convergent results. The energy profile is plotted in fig. 3(a), with $n$ up to 7, representing a 7-layer twin sandwiched between two untwinned crystal slabs.

We see from fig. 3(a) that unlike FCC metals, $\lambda=1$ in BCC Mo is not a metastable state but is unstable. This means there is no metastable 1-layer stacking fault in BCC Mo, so a full dislocation cannot split into partial dislocations with an extended 1-layer stacking fault between them. However, fig. 3(a) shows that the $\lambda=2$ state is metastable. A magnified view is given in fig. 3(b). This suggests that the smallest possible twin embryo in BCC Mo consists of 2 atomic layers. This assertion contradicts the result obtained using a pair potential [16], which states that at least 3 atomic layers are necessary to form a metastable twin. On the other hand, it agrees with calculations $[29,30]$ using the many-body Finnis-Sinclair empirical potential [31, 32]. To verify that $\lambda=2$ is indeed metastable, we again allow arbitrary in-plane relaxations for 

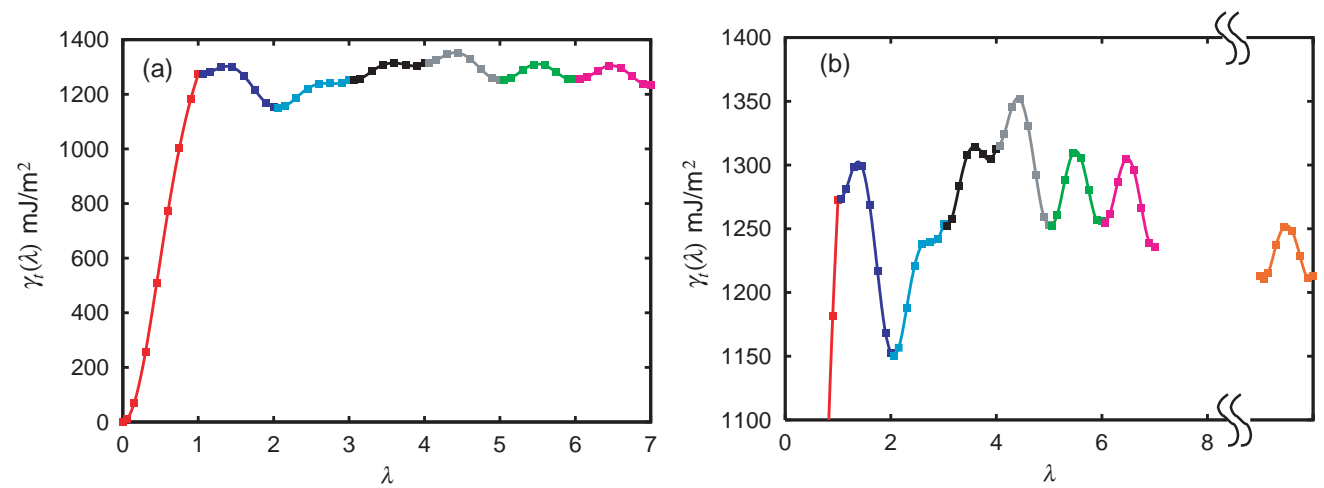

Fig. 3 - The $(2 \overline{1} \overline{1})\langle 111\rangle$ twinning energy pathway of BCC Mo up to 7-layer sliding using the 24-layer model. (b) is a magnified view of (a). The orange line and plots of the right edge of the graph are the energy landscape of twin boundary migration which is shifted twice the twin boundary formation energy of $607 \mathrm{~mJ} / \mathrm{m}^{2}$.

all 24 layers in VASP, until the energy change between two consecutive ionic steps is less than $1 \mathrm{meV}$. The unrelaxed vs. relaxed shear displacements of layers 8-17 are shown in fig. 4(a). We see that aside from a slight broadening, the $\lambda=2$ configuration is indeed locally stable.

Interestingly, the twin embryo, one layer thicker than the $\lambda=2$ metastable embryo, is again unstable. This is seen in fig. 3(b). To confirm this behavior, we allow in-plane relaxations with the ideal $\lambda=3$ initial configuration, to find that it spontaneously relaxes back to the $\lambda=2$ configuration.

The 4-layer embryo, $\lambda=4$, is a borderline case. From fig. 3(b), we see there is a small potential energy well at $\lambda=4$. But it is too weak to be trustworthy. Indeed, when we allow in-plane relaxations for all 24 layers in VASP, the $\lambda=4$ twin spontaneously relaxes back to the $\lambda=2$ configuration.

Finally, based on fig. 3(b) we believe that starting from $\lambda \geq 5$, every integer- $\lambda$ state is locally stable. The potential energy wells for $\lambda \geq 5$ appear to be too strong to be destabilized by relaxations, small applied stresses, and numerical error such as surface effects in the calculation. To verify this expectation, the unrelaxed $v s$. relaxed $\Delta \boldsymbol{x}_{i}$ 's of layers $8-17$ for $\lambda=5$ are shown in fig. $4(\mathrm{~b})$.
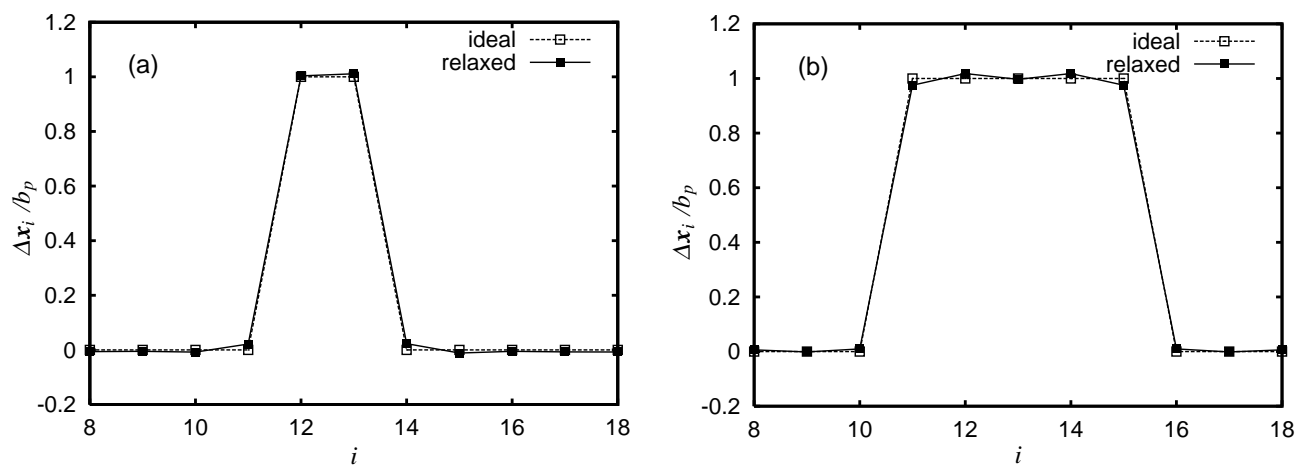

Fig. $4-\left\{\Delta \boldsymbol{x}_{i}\right\}$ (normalized by $\boldsymbol{b}_{\mathrm{p}}$ ) near the ideal and relaxed twin embryos. (a) $\lambda=2$, (b) $\lambda=5$. 
The consequence of the above results is that unlike in FCC metals [4], a twin embryo in BCC Mo can be nucleated only by the simultaneous emission of two tightly bound partial dislocations. Furthermore, this 2-layer twin embryo is far from mature. The next step in its evolution is to have an additional group of three tightly bound partial dislocations simultaneously nucleated. The Peierls-Rice-Tadmor framework $[4,33]$ with crack-tip shielding may still be applicable. However, one now needs to group the first two partial dislocations as A, and the next three partial dislocations as B, and take into account the A-B interactions and the detailed $\gamma_{\mathrm{t}}(\lambda)$ response. The mathematics will certainly be different from the FCC case. If one has to make an analogy with the FCC theory [4], then the effective " $\gamma_{\mathrm{ut}}$ " should be taken to be $\approx 1350-1150=200 \mathrm{~mJ} / \mathrm{m}^{2}$, which is the energy barrier between the $\lambda=2$ and the $\lambda=5$ energy minima (see fig. $3(\mathrm{~b})$ ).

From fig. 3, we see that starting from $\lambda=5$, the twin should be able to grow in a layerby-layer fashion. An interesting question is what is the core width of an isolated twin partial dislocation on a very thick deformation twin, when eq. (1) asymptote is applicable. Using the Peierls model [25], we estimate the FWHM of the Burgers vector density distribution (core width) of such partial dislocation (screw) to be $2 \xi=14.6 \AA$, which is very large. Then, using the discrete lattice sum scheme of Nabarro [26], we estimate the Peierls stress $\tau_{\mathrm{P}}$ is only $\sim 2 \mathrm{kPa}$ for the edge partial and $\sim 0.9 \mathrm{MPa}$ for the screw partial. Although the above calculation is non-variational [27], i.e. it assumes rigid core profile translation, we believe that in reality $\tau_{\mathrm{P}}$ 's are very small.

The small $\gamma_{\text {tbm }}$ also means that it is relatively easy to nucleate a twin partial on the boundary of a thick twin. The stress for athermal (spontaneous) nucleation of a twin boundary dislocation loop on a thick enough $(\lambda \geq 6)$ twin is only

$$
\tau_{\max }=\frac{\max _{\lambda}\left|\gamma_{\mathrm{tb} \infty}^{\prime}(\lambda)\right|}{\left|\boldsymbol{b}_{\mathrm{p}}\right|} \approx \frac{\pi \gamma_{\mathrm{tbm}}}{\left|\boldsymbol{b}_{\mathrm{p}}\right|} \approx 1.4 \mathrm{GPa},
$$

which is rather small, considering the low-temperature macroscopic critical resolved shear stress (CRSS) of BCC Mo is $750 \mathrm{MPa}$ [34]. In real materials, there are many local stress concentrators such as voids and interstitial clusters. Thus, once a thick enough deformation twin is formed, it should be quite easy for it to grow in thickness $[35,36]$ as it comes into contact with other inhomogeneities.

$$
* * *
$$

SO thanks H. Kitagawa and Y. ShiButani and acknowledges support by HattoriHoukoukai fellowship. JL and SY acknowledge support by Honda R\&D, AFOSR, DARPA, NSF, and LLNL.

\section{REFERENCES}

[1] Christian J. W. and Mahajan S., Prog. Mater. Sci., 39 (1995) 1.

[2] Chen M. W., Ma E., Hemker K. J., Sheng H. W., Wang Y. M. and Cheng X. M., Science, 300 (2003) 1275.

[3] Meyers M. A., Vohringer O. and Lubarda V. A., Acta Mater., 49 (2001) 4025.

[4] Tadmor E. B. and Hai S., J. Mech. Phys. Solids, 51 (2003) 765.

[5] Bernstein N. and Tadmor E. B., Phys. Rev. B, 69 (2004) 094116.

[6] Kaspar J., Luft A. and Skrotzki W., Cryst. Res. Technol., 35 (2000) 437.

[7] Agnew S. R. and Leonhardt T., JOM-J. Miner. Met. Mater. Soc., 55 (2003) 25.

[8] Cerv J., Landa M. and Machova A., Scr. Mater., 43 (2000) 423. 
[9] Cottrell A. H. and Bilby B. A., Philos. Mag., 42 (1951) 573.

[10] Sleeswyk A. W., Philos. Mag., 29 (1974) 407.

[11] Mahajan S., Acta Metall., 23 (1975) 671.

[12] Pirouz P., Scr. Metall., 21 (1987) 1463.

[13] Lagerlof K. P. D., Acta Metall. Mater., 41 (1993) 2143.

[14] Frenkel J., Z. Phys., 37 (1926) 572.

[15] Vitek V., Philos. Mag., 18 (1968) 773.

[16] Vitek V., Scr. Metall., 4 (1970) 725.

[17] Ogata S., Li J. and Yip S., Science, 298 (2002) 807.

[18] Ogata S., Li J. and Yip S., Phys. Rev. B, to be submitted (2004).

[19] Kresse G. and Furthmüller J., Phys. Rev. B, 54 (1996) 11169.

[20] Perdew J. P. Chevary J. A., Vosko S. H., Jackson K. A., Pederson M. R., Singh D. J. and Fiolhais C., Phys. Rev. B, 46 (1992) 6671.

[21] Vanderbilt D., Phys. Rev. B, 41 (1990) 7892.

[22] Methfessel M. and Paxton A. T., Phys. Rev. B, 40 (1989) 3616.

[23] Landolt-Börnstein, Vol. III/29a (Springer-Verlag, Heidelberg) 1992.

[24] Krenn C. R., Roundy D. and Morris J. W. jr. and Cohen M. L., Mater. Sci. Eng. A, 319 (2001) 111.

[25] Peierls R., Proc. Phys. R. Soc. London, 52 (1940) 34.

[26] Nabarro F. R. N., Proc. Phys. R. Soc. London, 59 (1947) 256.

[27] Bulatov V. V. and Kaxiras E., Phys. Rev. Lett., 78 (1997) 4221.

[28] Mrovec M. et al., Z. Metallk., 94 (2003) 244.

[29] Chang J.-P., PhD Thesis, Massachusetts Institute of Technology (2002).

[30] Xu D.-S., Chang J.-P., Li J., Yang R., Li D. and YiP S., to be published in Mater. Sci. Eng. A (2004).

[31] Finnis M. W. and Sinclair J. E., Philos. Mag. A, 50 (1984) 45.

[32] Finnis M. W. and Sinclair J. E., Philos. Mag. A, 53 (1986) 161.

[33] Xu G., Argon A. S. and Ortiz M., Philos. Mag. A, 72 (1995) 415.

[34] Kaufmann H.-J., Luft A. and Schulze D., Cryst. Res. Technol., 19 (1984) 357.

[35] Sumino K., Acta Metall., 14 (1966) 1607.

[36] Suezawa M. and Sumino K., Phys. Status Solidi A, 36 (1976) 263. 